\title{
Salvage Transoral Robotic Surgery for Recurrent or Residual Head and Neck Squamous Cell Carcinoma: A Single Institution Experience
}

\author{
Surender Dabas ${ }^{1 \&}$, Abhinav Dewan ${ }^{2 *}$, Reetesh Ranjan ${ }^{1}$, Ajay Kumar Dewan ${ }^{1}$, \\ Himanshu Shukla ${ }^{1}$, Rupal Sinha ${ }^{3}$
}

\begin{abstract}
Background: The present study was conducted to evaluate the technical feasibility, safety and adequacy of surgical margins with salvage transoral robotic surgery (TORS) for recurrent or residual head and neck squamous cell carcinoma patients. Materials and Methods: Thirty patients who underwent salvage TORS using the 'DaVinci' robot were enrolled in the study and data related to their surgical time, complications and functional outcome were recorded. Results: The feasibility of salvage TORS in our study was observed to be $100 \%$. Positive margins were encountered in only $6.7 \%$ of patients. Mean blood loss was $23.3 \mathrm{ml}$ with no patient requiring blood transfusion. Postoperative complications in the form of primary haemorrhage requiring active surgical intervention occurred in $13.3 \%$. Oral feeding could be started as early as the 3rd postoperative day in a few patients, with nasogastric tubes being removed on the 12th postoperative day. Long term gastrostomy tube dependency was seen in $10 \%$ cases. Median survival of patients was 19 months. Conclusions: Salvage TORS is a safe, effective and feasible option in the management of treatment failure cases. It offers an alternative surgical approach with unexpected benefits in terms of tracheostomy tube use, Ryle's tube and gastrostomy dependence.
\end{abstract}

Keywords: Salvage TORS - recurrent or residual head neck cancer - feasability - safety

Asian Pac J Cancer Prev, 16 (17), 7627-7632

\section{Introduction}

Historically, management of head and neck squamous cell carcinoma (HNSCC) required extensive open surgical resection followed by adjuvant therapy. This approach resulted in considerable functional morbidity and poor cosmetic outcomes. However, recently there has been a paradigm shift in management of HNSCC with increasing focus on organ preservation strategies like chemoradiation. Chemoradiation is becoming more prevalent and provides survival benefit comparable to surgery. Gradually, surgery is being reserved for salvage treatment in case of recurrence after primary therapy based on radiotherapy (Chen et al., 2007).

Although chemoradiation shows excellent local control, HNSCC patients are at increased risk of recurrence after initial therapy (Roosli et al., 2010). Recurrent cases are considered highly aggressive, infiltrative, technically challenging and have a dismal prognosis, irrespective of the fact whether relapse occurred in the primary site or in the neck (Richey et al., 2007; Roosli et al., 2010). Treatment of such cases has most commonly been palliative in nature. Salvage surgery remains the only curative treatment option for patients with failure after radiotherapy (Rate et al., 1991). Recurrent or residual cases were traditionally managed through open surgical approaches that were functionally debilitating, resulting in decrease in quality of life (QOL). Concerns regarding QOL, poor surgical outcome and technical challenges associated with salvage surgery led many authors to conclude against using salvage surgery for treatment failure cases. Hence, the decision to perform salvage surgery remains controversial.

However, the recent approach to decrease this surgical morbidity and improve functional and surgical outcomes has been the use of minimally invasive surgical procedures like transoral robotic surgery (TORS). In contrast to the traditional open surgical approaches, TORS provides excellent oncological outcomes with fewer complications and an improved speech and swallowing function (Genden et al., 2009; Moore et al., 2009; Weinstein et al., 2009). TORS has simplified the surgical approach by providing excellent tumour visualization and it allows for conversion of fine finger movement of surgeon into precise movement of robotic arms within narrow confines of head and neck. Additional benefits include decreased hospital stay, high rates of negative surgical margin and better cosmesis (Boudreaux et al., 2009; Genden et al., 2009; Moore et 
al., 2009; White et al., 2013). Careful patient selection is essential to achieve precise balance of QOL, reduction of post operative complications and improved survival. Inspite of low tumor control probability, salvage TORS can be offered as a valuable option in carefully selected cases.

The present study was designed to evaluate the technical feasibility, safety and adequacy of surgical margins with salvage TORS in recurrent or residual HNSCC patients managed initially with radiotherapy or chemoradiotherapy.

\section{Materials and Methods}

Between March'2013 and December'2014, thirty patients with biopsy-proven recurrent or residual HNSCC underwent robot assisted surgical resection using 'DaVinci robot' in a prospective observational study conducted at Rajiv Gandhi Cancer Institute and Research Centre, Delhi after approval from institutional review board. Written informed consent was taken from patients who met the inclusion and exclusion criteria. Inclusion criteria for the study comprised of patients $>18$ years age with recurrent or residual HNSCC following definitive radiation with or without chemotherapy. Contraindications of salvage TORS included patients who failed to provide written consent, had inadequate mouth opening $(<1.5 \mathrm{~cm})$, presence of AJCC TNM rcT4b stage (Recurrent clinical stage T4b), invasion of deep tissue lateral to constrictor muscle or posterior invasion of deep vertebral fascia, unresectability of involved lymphnodes, distant metastasis, medical contraindications for general anaesthesia and surgery and unexplained active infection.

The flow of patients enrolled in the present study is shown in Figure 1. Patients were evaluated for age, sex, primary tumor location, previous treatment, histopathological features, management of neck disease, perioperative complications, functional and surgical outcomes. Study endpoints included feasibility, safety and adequacy of surgical margins. Feasibility was defined in terms of ability to perform salvage TORS successfully without the need of intraoperative conversion to open procedure. Safety was evaluated by the rate of perioperative complications and functional outcomes, including the percutaneous endoscopic gastrostomy (PEG), Ryle's tube and tracheostomy tube dependency. Adequacy of surgical margins was determined by the presence of positive margin on final histopathology report. Variability in definition of close margin is present from institution to institution. At University of Pennysylvania and Mayo's, a margin of $<2 \mathrm{~mm}$ is considered close. We have considered a similar margin of $2 \mathrm{~mm}$ as cut-off value between close and free in our study (Weinstein et al., 2012).

Neck dissection was performed when indicated as a concomitant procedure. In the present study, patients underwent either unilateral or bilateral modified neck dissection in case of clinical or radiological nodal positivity. Neck was not addressed in case of node negative neck. Diagnostic workup prior to surgical treatment included clinical examination, pan-endoscopy, tumor biopsy and radiological examination (MRI/PET scan). Patients were evaluated by means of clinical examination every month for 3 months, followed by repeat examination every 3 monthly by the surgical team. Follow up information including details of recurrence and survival status were recorded for every patient. All statistical analysis was performed by the standard methods using SPSS computer software (Version 16, SPSS Inc, Chicago, IL, USA).

\section{Results}

\section{Patient and tumor characteristics}

Thirty patients with HNSCC who had residual or recurrent disease following radiotherapy underwent salvage TORS procedure (Figure 2 and 3). Patient and tumor characteristics are summarized in Table 1. Average age of subjects was $56.8 \pm 11.3$ years (range $31-86$ years) and majority were males $(96.7 \%)$. All the patients had Charlson Comorbidity index of 0-5. The commonest site of tumor location was oropharynx $(n=26,86.7 \%)$. Based on pre-chemoradiation staging, four patients had T1 primary tumor, 15 had T2, 5 had T3 and 6 had T4 tumor stage. The rates for lymph node metastasis were $50 \%$ with $\mathrm{T} 1,66.7 \%$ with $\mathrm{T} 2,40 \%$ with $\mathrm{T} 3$ and $83.3 \%$ with T4 disease. Of the 30 patients, $8(26.7 \%)$ received radical RT alone (66-70Gy/33-35\#), while 22 (73.3\%) patients received concurrent chemoradiation $(70 \mathrm{~Gy} / 35 \#)$. Patients were administered weekly concurrent chemotherapy with injection cisplatin $35-40 \mathrm{mg} / \mathrm{m} 2$. When local or regional recurrence was detected during the follow up after initial treatment, appropriate evaluation and surgical treatment

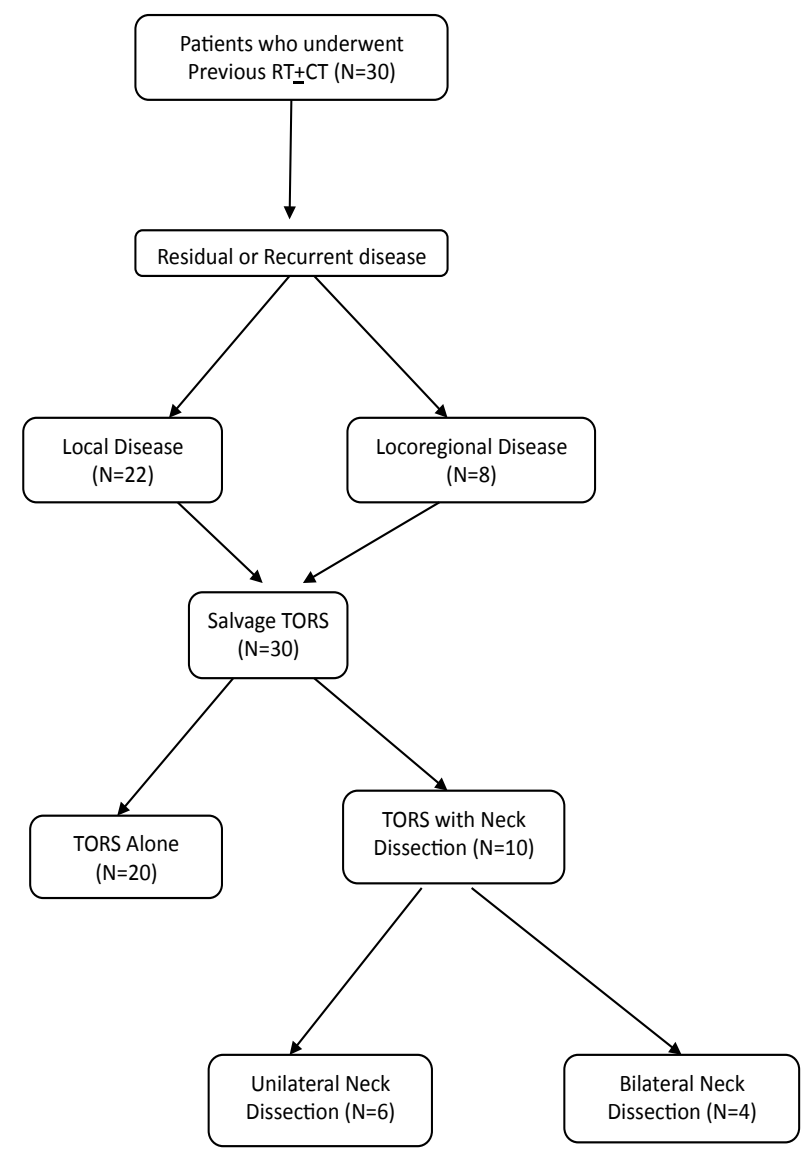

Figure 1. Flow of Patients Enrolled in the Present Study 
was conducted. Majority of the patients $(73.3 \%)$ had local recurrence alone, while remaining patients had locoregional recurrence $(26.7 \%)$.

There was $100 \%$ feasibility for salvage TORS in the present study. Simultaneous unilateral $(n=6)$ or bilateral neck dissection $(n=4)$ was performed in 10 $(33.3 \%)$ patients. Majority of the patients $(n=26)$ had moderately differentiated squamous cell carcinoma on final histopathology report. Two patients (pT0) with biopsy proven residual/recurrent disease had no evidence of malignancy on final histopathology report. Pathological $\mathrm{T}$ and $\mathrm{N}$ stage of salvage TORS patients are shown in Table 2. Seven patients had evidence of extracapsular extension in neck dissection specimen. There was presence of certain other adverse pathological features such as lymphovascular and perineural invasion in 7 (23.33\%) and $12(40 \%)$ patients, respectively. No postoperative treatment was recommended and all patients were kept on close follow up.

\section{Assessment of surgical outcome}

Operative and postoperative data including blood loss, operative time and margin status were recorded

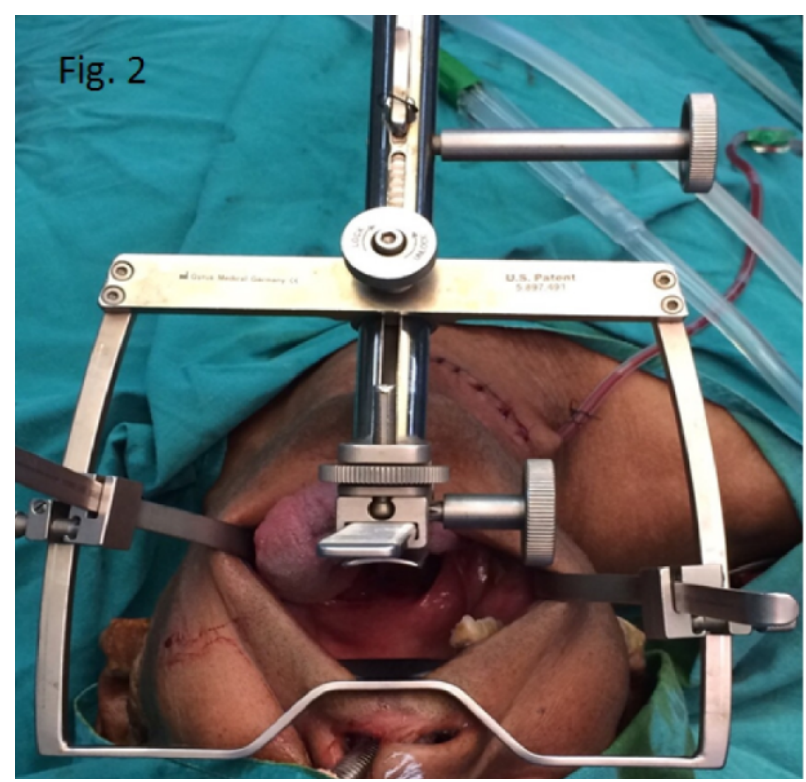

Figure 2. Transoral Robotic Surgery setup - Intraoral Exposure obtained via Feyh-Kastenbauer retractor (FK retractor)
DOI:http://dx.doi.org/10.7314/APJCP.2015.16.17.7627

Salvage TORS for Recurrent or Residual Head Neck Cancer

and analysed. Of the 30 patients, positive and close surgical margin was reported in $6.7 \%$ cases each, thereby demonstrating adequacy of surgical margins for patients undergoing TORS. Estimated blood loss during TORS was $23.3 \pm 20.1 \mathrm{ml}$ (range $5-100 \mathrm{ml}$ ), with no patient requiring blood transfusion. Average operating time for TORS procedure (without neck dissection), standard setup time and robotic setup time was $62.3 \pm 24.5$ minutes (range, 20-120 minutes), 7.7 \pm 9.4 minutes (Range, 1.50-31 minutes) and 5.71 \pm 4.74 minutes (range, 1.5-21 minutes) respectively. Standard setup, robotic setup and surgical time decreased dramatically as the operating room staff and physicians gained more experience with daVinci robotic surgical system.

None of the patients had intraoperative or perioperative fatalities. Of the 30 patients undergoing TORS, four patients developed postoperative bleeding requiring active surgical intervention. All the bleeding episodes occurred in first postoperative week. One patient who had undergone TORS alongwith simultaneous neck dissection developed pharyngo-cutaneous fistula that was closed with sternocleidomastoid buttressing. Nasal twang was reported in $10 \%$ patients on long term follow up. Aspiration pneumonia occurred in one patient who underwent transoral robotic supraglottic laryngectomy and was managed with emergency tracheostomy.

\section{Functional outcome}

Functional outcome was defined in terms of hospital stay, tracheostomy dependency, duration of Ryle's tube

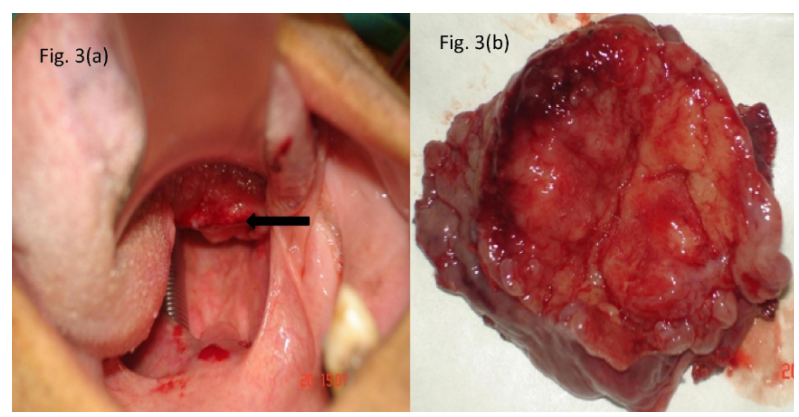

Figure 3. (a) Intraoperative presurgical view of patient with rcT2 base of tongue squamous cell carcinoma lesion (marked by an arrow sign); (b) Transoral robotic surgical specimen for histopathological assessment

\section{Table 1. Patient and Tumor Characteristics}

\begin{tabular}{ll}
\hline Characteristics & \multicolumn{1}{c}{ Number of Patients } \\
\hline Age (Mean + standard deviation) & $56.80 \pm 11.34$ years (Range, 31-86 years) \\
Sex (Male/Female) & $29 / 1(96.7 \% / 3.3 \%)$ \\
Previous treatment (RT alone/ Concurrent chemoradiation) & $8(26.7 \%) / 22(73.3 \%)$ \\
Smoker (Yes/ No) & $26(86.7 \%) / 4(13.3 \%)$ \\
Alcoholic (Yes/ No) & $6(20 \%) / 24(80 \%)$ \\
Tobacco chewer (Yes/ No) & $7(23.3 \%) / 23(76.7 \%)$ \\
Primary Tumor Location (Hypopharynx/ Larynx/ Oropharynx) & $1(3.3 \%) / 3(10 \%) / 26(86.7 \%)$ \\
Post op Histology (Mod diff / Poorly diff /No Evidence) & $26(86.7 \%) / 2(6.7 \%) / 2(6.7 \%)$ \\
Pathological T stage (T0/T1/T2/T4) & $2(6.7 \%) / 10(33.33 \%) / 14(46.7 \%) / 4(13.3 \%)$ \\
Pathological N stage (NX/N0/N1/N2b/N2c) & $20(66.7 \%) / 3(10 \%) / 1(3.3 \%) / 5(16.7 \%) / 1(3.3 \%)$ \\
Overall Pathological Stage (I/II/II//V/X) & $9(30 \%) / 10(33 \%) / 1(3.3 \%) / 9(30 \%) / 1(3.3 \%)$ \\
\hline
\end{tabular}


Table 2. Pathological TNM Staging of Patients who Underwent Salvage TORS

T-Classification Number of Patients by N-Classification

\begin{tabular}{lcccccc} 
& pNX & pN0 & pN1 & pN2b & pN2c & Total \\
\hline pT0 & 1 & 0 & 1 & 0 & 0 & 2 \\
pT1 & 8 & 1 & 0 & 1 & 0 & 10 \\
pT2 & 8 & 2 & 0 & 3 & 1 & 14 \\
pT4 & 3 & 0 & 0 & 1 & 0 & 4 \\
Total & 20 & 3 & 1 & 5 & 1 & 30 \\
\hline
\end{tabular}

and PEG use. Mean duration of hospital stay was $5.9 \pm 6.6$ days. Patients tolerated the TORS procedure well, with oral feeding being started as early as 3rd post operative day in a few patients and patients resuming normal diet after a mean duration of 20.2 days. Average duration of Ryles use was $12.9 \pm 9.5$ days. PEG was inserted in $5(16.7 \%)$ patients during surgery, and was removed after 30 days of use in 4 patients. Long term PEG dependency was reported in 3.3\% patients only. Long term tracheostomy tube dependence was seen in $10 \%$ cases.

\section{Survival and Oncological Outcome}

Out of the 30 patients enrolled in the study, 3 patients died $(10 \%)$. One patient died of non-oncological cause. The overall survival of the patients was $86 \%$ at 122 months. The median survival of the patients was 19 months (7-122 months). Time to recurrence was calculated as the time period between the time of salvage TORS and recurrence. A total of 13 patients (43.3\%) had recurrence. The median time to recurrence was 3 months (range 1-17 months).

Disease control was achieved in 17 patients (Successful salvage rate $56.7 \%$ ). Almost $57.7 \%$ and $66.67 \%$ patients with tumors in oropharynx and larynx respectively were successfully salvaged by TORS. Salvage rates decreased with increase in pathological T- stage (pT0$50 \%$, pT $1-60 \%$, pT2-64.3\%, pT3-0\%, pT4-25\%). The recurrence pattern after salvage TORS was as follows: Local recurrence $(30 \%)$, regional recurrence $(3.3 \%)$, loco-regional recurrence $(6.7 \%)$ and distant metastasis (3.3\%). These cases were then put on either palliative chemotherapy or targeted therapy. One patient was operated for regional recurrence and underwent reirradiation for the same. Three patients developed a second primary tumor in oral cavity and underwent another open surgical procedure for the same. Salvage TORS for laryngeal and hypopharyngeal tumors allowed preservation of larynx in all $13.33 \%(n=4)$ cases.

\section{Discussion}

The present study documents the feasibility, safety and adequacy of surgical margins of salvage TORS for the treatment of recurrent or residual head and neck cancers. To our knowledge, there has been one retrospective multi-institutional study by White et al. (2013) comparing oncological and functional outcomes of patients with recurrent oropharyngeal cancer treated by TORS with that of an open surgery. Our study is a prospective observational single institution study that shares our initial experience with salvage TORS in recurrent head and neck cancer setting and compares our data with White et al. (2013) and other historical data.

The feasibility of TORS was clearly demonstrated; $100 \%$ patients were salvaged with TORS without the need of intraoperative conversion to open surgery. In another multi-institutional study, Weinstein et al. (2012) assessed feasibility and safety of TORS procedure for head and neck cancer cases (including naive and salvage cases). Most patients (98.9\%) in this study successfully underwent TORS without intraoperative conversion to open procedure. In contrast, in a study of nonrobotic transoral surgical resection of tonsillar tumors by Moore et al. (2009), 5.9\% (6/102) subjects required conversion to an open procedure. Another study by Preuss et al. (2009) reported 3.6\% intraoperative conversion rates for laryngeal cancer patients undergoing transoral laser resection. Thus, resection of head and neck tumors by TORS may result in decreased intraoperative conversion as compared to resection by conventional transoral approaches even in salvage cases.

Surgical Outcomes: Mean blood loss reported in our study was $23.3 \pm 20.1 \mathrm{ml}$, with no patient requiring blood transfusion. White et al. (2013) reported a low incidence of estimated blood loss $(49 \mathrm{ml})$ in salvage TORS arm (Blood loss in open surgery arm $331 \mathrm{ml}, \mathrm{p}<0.001$ ). Operative and setup time, as an indicator of surgical efficacy, may be influenced by multiple factors, including surgeon experience, tumour size, site, previous history of irradiation etc and is considered to be a cost-effective and a safe indicator. TORS procedure has a sharp learning curve and a precipitous decline in standard setup, robotic setup and surgical time with increase in experience was reported in our study. Other studies (2013) have also reported a low mean operative time in salvage TORS arm in comparison to open salvage surgical arm for recurrent oropharyngeal cancers (111 minutes vs 350 minutes, $\mathrm{p}<0.001$ ). TORS thus allows for shorter operating time than open surgery, which could be beneficial for elderly patients.

Assessment of surgical margins: The overall incidence of positive and close tumor margins in our study was $6.7 \%$ each. Positive margins were reported in 2 recurrent oropharyngeal carcinoma cases, while close margin was reported in 1 case each of hypopharyngeal and oropharyngeal cancer after TORS resection. None of the patients with laryngeal cancer had positive or close margins following TORS resection. These results compare favourably with other salvage TORS and open surgical studies. White et al. (2013) compared TORS and open surgical approach for salvage surgery in recurrent oropharyngeal cancers. A positive surgical margin rate of $9 \%$ in the TORS group and $29 \%$ in the open surgery group ( $\mathrm{p}=0.007$ ) was observed. Thus, in terms of adequacy of surgical margins, salvage TORS appears significantly superior to open surgical approaches.

Safety of TORS procedures: Our study reported an acceptable frequency of post operative complications after radiation. There were no intraoperative or perioperative fatalities in our study. The incidence of postoperative transoral bleeding requiring active intervention in this 
study was $13.3 \%$, which was lower than that reported by White et al. (2013) (21.9\%) in the salvage TORS arm. None of the patients in our study had carotid artery injury as compared to $1.2-3 \%$ reported after open procedures or radiation (Ketcham and Hoye, 1965; Shumrick DA, 1973; Joseph and Shumrick, 1973; Sobol et al., 1982; Jackel et al., 2007). Wound dehiscence did not occur in any patient compared to 2-14.3\% reported after open surgery (O'Brien et al., 1993; Weber et al., 2003).

One patient $(3.3 \%)$ developed pharyngocutaneous fistula that was salvaged with sternocleidomastoid buttressing in our study. Higher incidence of fistula has been reported in patients who are post radiotherapy and those who have undergone simultaneous neck dissection in comparison to naive patients. None of the patients in the salvage TORS arm developed pharyngocutaneous fistula in the study by White et al. (2013). Clark et al. (2006) showed that radiation increased the incidence of post operative pharyngocutaneous fistula rates from 24 to $38 \%$, while Wakisaka et al. (2008) noted delayed fistula closure after radiotherapy.

Functional Outcomes:TORS allows for organ preservation and a better functional rehabilitation with decreased morbidity. Functional outcomes were defined in terms of PEG dependency, duration of Ryle's tube usage, speech outcome, tracheostomy tube dependence and hospital stay.

Historical data indicates that very few patients $(8 \%)$ who undergo salvage surgery for oropharyngeal cancers return to normal diet and majority remain dependent on feeding tube (56\%) (Kostrzewa et al., 2010). In our study, PEG tube and Ryle's tube dependency was used as a surrogate indicator for the preservation of swallowing function. Ryle's tube was inserted in 25 patients during surgery and removed after a mean duration of 12.9 days. PEG insertion was done in 5 cases during surgery in our study. Long term PEG dependency rates $(3.3 \%)$ in our study were comparable to that reported by White et al. (2013) for recurrent oropharyngeal cancer cases. Thirty five percent patients in the salvage TORS arm and $75 \%$ cases in open surgical arm had feeding tube at time of surgery. On 1 year follow up, 3\% patients in salvage TORS group still required feeding tube support. Normal diet was resumed on 20th postoperative day in majority of our patients, thereby indicating rapid swallowing rehabilitation.

Long term tracheostomy tube dependence was reported in $10 \%$ cases in the present study. White et al. (2013) compared functional outcomes in salvage TORS vs open salvage surgery arm for recurrent oropharyngeal cancers and reported higher incidence of tracheostomy tube insertion in open surgery arm at the time of surgery (79\% vs $23 \%, \mathrm{p}<0.01)$. Majority of the patients in the TORS arm were decannulated in 1-2 weeks. Most patients in the open salvage surgical arm required either temporary or permanent tracheostomy in the above study. Our results compared favourably with other salvage TORS studies and incidence of tracheostomy tube dependence was significantly lower in comparison to previously published open salvage surgical studies (O'Brien et al., 1993; Weber et al., 2003; Clark et al., 2006).
Nasal twang following TORS was noted in $3(10 \%)$ patients in the immediate postoperative period. Similar trend was seen by White et al. (2013) who reported improved speech outcome in salvage TORS arm in comparison to open salvage surgical arm. Rapid speech and swallowing rehabilitation was accompanied by a shorter hospital stay. The mean hospital stay of our patients was shorter than that of patients who would have otherwise undergone an open surgery. Mean duration of hospital stay was $5.9 \pm 6.6$ days in our study. On comparing salvage TORS and open surgery arm, a significant difference in mean hospital stay was seen ( 8 vs 3.8 days, $\mathrm{p}<0.001$ ) in another study (White et al., 2013).

Oncological outcomes: Recurrent HNSCC is associated with poor overall survival rates. Surgical salvage, however, may be the only option at potential cure for the patient. However, survival even with salvage surgery tends to be disappointingly poor (Wakisaka et al., 2008; Boudreaux et al., 2009; Kostrzewa et al., 2010). Role of salvage surgery in oropharyngeal cancer has been studied and reported by Bachar et al. (2010) and Zafereo et al. (2009). In the study conducted by Bachar et al. (2010) during 1970-90's, 175 tonsillar cancer patients were initially treated with primary radiotherapy and managed with surgical salvage for locoregional recurrence. Bachar et al. reported a 5 year overall and cause specific survivals of $23 \%$ and $40 \%$ respectively. Zafereo et al. (2009) described the outcome of 168 oropharyngeal cancer patients with local recurrence treated initially with radiation or chemoradiation. Forty one of these underwent salvage surgery and a 5-year OS of $28 \%$ was reported. All these studies used open surgical approaches leading to functional sacrifice and diminished QOL for patients.

Surgical salvage using TORS seem to be a reasonable alternative to open surgical procedures for recurrent/ residual head and neck cancer cases. It has been shown to be associated with acceptable limited morbidity, low rates of PEG and tracheostomy dependence, shorter operative time, minimal blood loss and shorter hospital stay. Also, it may be essential to compare survival rates and functional outcomes of any new proposed treatment option (such as TORS in our case) with current known rates. White et al. (2013) demonstrated a significant difference in survival between the TORS and open surgical approach (2 year OS - $74 \%$ vs $43 \%$, p <0.05). This may have been due to higher positive margins reported in the open surgical arm. Median survival in our study with TORS was 19 months. Overall, a total of $56.7 \%$ patients were successfully salvaged with TORS. The median time to recurrence in our study was 3 months. However, a longer follow up is required to comment further on the long term functional outcome and disease control.

Limitations of our study included nonrandomization of our patients, short duration of follow up, small patient number, validated questionnaires required to assess speech and swallowing function not available and absence of long term survival data. However, this study represents a unique single institution experience in India of salvage TORS in recurrent or residual head and neck cancer setting. To our knowledge, there has not been any published prospective study addressing the clinical course of patients, their 


\section{Surender Dabas et al}

surgical and functional outcomes and complications with salvage TORS after treatment failure. Further prospective studies examining the role of salvage TORS in recurrent or residual setting are warranted to determine if this technique provides an advantage over currently available treatment modalities.

Salvage TORS represents a new minimally invasive approach in head and neck surgery and is a safe, feasible, and effective option for management of the treatment failures cases.

\section{References}

Bachar GY, Goh C, Goldstein DP, et al (2010). Long term outcome analysis after surgical salvage for recurrent tonsil carcinoma following radical radiotherapy. Eur Arch Otorhinolaryngol, 267, 295-301.

Boudreaux BA, Rosenthal EL, Magnuson JS, et al (2009). Robotassisted surgery for upper aerodigestive tract neoplasms. Arch Otolaryngol Head Neck Surg, 135, 397-401.

Chen AY, Schrag N, Hao Y, et al (2007). Changes in treatment of advanced oropharyngeal cancer, 1985-2001. Laryngoscope, 117, 16-21.

Clark JR, De Almedia J, Gilbert R, et al (2006). Primary and salvage (hypo) pharyngectomy: analysis and outcome. Head Neck, 28, 671-7.

Genden EM, Desai S, Sung CK (2009). Transoral robotic surgery for the management of head and neck cancer: a preliminary experience. Head Neck, 31, 283-9.

Genden EM, Desai S, Sung CK (2009). Transoral robotic surgery for the management of Head Neck, 31, 283-9.

Jackel MC, Ambrosch P, Martin A, et al (2007). Impact of reresection for inadequate margins on the prognosis of upper aerodigestive tract cancer treated by laser microsurgery. Laryngoscope, 117, 350-6.

Joseph DL, Shumrick DL (1973). Risks of head and neck surgery in previously irradiated patients. Arch Otolaryngol, 97, 381-4.

Ketcham AS, Hoye RC (1965). Spontaneous carotid artery hemorrhage after head and neck surgery. Am J Surg, 110, 649-55.

Kostrzewa JP, LancasterWP, Iseli TA, et al (2010). Outcomes of salvage surgery with free flap reconstruction for recurrent oral and oropharyngeal cancer. Laryngoscope, 120, 267-272.

Moore EJ, Henstrom DK, Olsen KD, et al (2009). Transoral resection of tonsillar squamous cell carcinoma. Laryngoscope, 119, 508-15.

Moore EJ, Olsen KD, Kasperbauer JL (2009). Transoral robotic surgery for oropharyngeal squamous cell carcinoma: A prospective study of feasibility and functional outcomes. Laryngoscope, 119, 2156-64.

Moore EJ, Olsen KD, Kasperbauer JL (2009). Transoral robotic surgery for oropharyngeal squamous cell carcinoma: a prospective study of feasibility and functional outcomes. Laryngoscope, 119, 2156-64.

O'Brien CJ, Nettle WJ, Lee KK (1993). Changing trends in the management of carcinoma of the oral cavity and oropharynx. Aust N Z J Surg, 63, 270-4.

Preuss SF, Cramer K, Klussmann JP, et al (2009). Transoral laser surgery for laryngeal cancer: outcome, complications and prognostic factors in 275 patients. Eur J Surg Oncol, 35, 235-40.

Rate WR, Garrett P, Hamaker R, et al (1991). Intraoperative radiation therapy for recurrent head and neck cancer. Cancer, 67, 2738-40.

Richey LM, Shores CG, George J, et al (2007). The effectiveness of salvage surgery after the failure of primary concomitant chemoradiation in head and neck cancer. Otolaryngol Head Neck Surg, 136, 98-103.

Roosli C, Studer G, Stoeckli SJ (2010). Salvage treatment for recurrent oropharyngeal squamous cell carcinoma. Head Neck, 32, 989-96.

Shumrick DA (1973). Carotid artery rupture. Laryngoscope, 83, 1051-61.

Sobol SM, Freeman R, Thawley S, et al (1982). Management of inadvertent injury to the carotid artery during head and neck surgery. Head Neck Surg, 4, 475-82.

Wakisaka N, Murono S, Kondo S, et al (2008). Post operative pharyngocutaneous fistula after laryngectomy. Auris Nasus Larynx, 35, 203-208.

Weber RS, Berkey BA, Forastiere A, et al (2003). Outcome of salvage total laryngectomy following organ preservation therapy: the Radiation Therapy Oncology Group trial 91-11. Arch Otolaryngol Head Neck Surg, 129, 44-49.

Weinstein GS, O’Malley BW Jr, Desai SC, et al (2009). Transoral robotic surgery: Does the ends justify the means? Curr Opin Otolaryngol Head Neck Surg, 17, 126-131.

Weinstein GS, O’Malley BW Jr, Magnuson JS, et al (2012). Transoral robotic surgery: a multicenter study to assess feasibility, safety, and surgical margins. Laryngoscope, 122, 1701-7.

White H1, Ford S, Bush B, et al (2013). Salvage surgery for recurrent cancers of the oropharynx: comparing TORS with standard open surgical approaches. JAMA Otolaryngol Head Neck Surg, 139, 773-8.

Zafereo ME, Hanasono MM, Rosenthal DI, et al (2009). The role of salvage surgery in patients with recurrent squamous cell carcinoma of the oropharynx. Cancer, 115, 5723-33. 